\title{
Asiaticoside produces an antidepressant-like effect in a chronic unpredictable mild stress model of depression in mice, involving reversion of inflammation and the PKA/pCREB/BDNF signaling pathway
}

\author{
LUOQING WANG $^{1 *}$, TING GUO ${ }^{2 *}$, YUANFANG GUO ${ }^{3 *}$ and YUJIE XU \\ ${ }^{1}$ Department of Cardiovascular Medicine, The Second People's Hospital of Lianyungang, Lianyungang, Jiangsu 222000; \\ ${ }^{2}$ Department of Neurology, Xuzhou Medical University Affiliated Hospital of Lianyungang, Lianyungang, Jiangsu 222061; \\ ${ }^{3}$ Department of Respiratory Medicine, Ganyu District People's Hospital, Lianyungang, Jiangsu 222100; ${ }^{4}$ Department of \\ Anesthesiology and Perioperative Medicine, Jiangsu Province Hospital, Nanjing, Jiangsu 210000, P.R. China
}

Received July 9, 2019; Accepted May 4, 2020

DOI: $10.3892 / \mathrm{mmr} .2020 .11305$

\begin{abstract}
Asiaticoside is one of the triterpenoid components found in Centella asiatica that has promising neuroprotective properties. The present study aimed to evaluate the antidepressant-like properties of asiaticoside and to investigate the possible mechanisms underlying its mode of action using a mouse model of chronic unpredictable mild stress (CMS). Behavioral tests, including sucrose preference test, forced swimming test and tail suspension test, were performed to evaluate symptoms of depression. The expression levels of neurotransmitters, 5-hydroxytryptamine (5-HT) and norepinephrine $(\mathrm{NE})$, in the hippocampus were measured by high-performance liquid chromatography. ELISA and western blotting were used to detect protein expression. It was demonstrated that asiaticoside treatment (20 and 40 $\mathrm{mg} / \mathrm{kg}$; intragastric) significantly reversed the decrease in sucrose consumption, and reduced the immobility time in tail suspension tests and forced swimming tests in CMS mice. Furthermore, asiaticoside treatment upregulated the expression of 5-HT and NE in the CMS mouse model. Asiaticoside administration also downregulated the levels of interleukin (IL)-1 $\beta$, IL- 6 and tumor necrosis factor- $\alpha$ in the hippocampus, and reduced the phosphorylation of nuclear factor (NF)- $\kappa$ Bp65 and the expression of nod-like receptor protein 3 (NLRP3), thus
\end{abstract}

Correspondence to: Dr Yujie Xu, Department of Anesthesiology and Perioperative Medicine, Jiangsu Province Hospital, 300 Guangzhou Road, Nanjing, Jiangsu 210000, P.R. China

E-mail: xyj3196@163.com

*Contributed equally

Key words: asiaticoside, inflammatory cytokines, phosphorylated $\mathrm{NF}-\kappa \mathrm{Bp} 65$, nod-like receptor protein 3 inflammasome, cAMP/ protein kinase $\mathrm{A}$ decreasing the expression of mature caspase-1. Furthermore, asiaticoside significantly increased the levels of cAMP and protein kinase A (PKA), and enhanced phosphorylation of the cAMP-related specific marker vasodilator-stimulated phosphoprotein at serine 157 . Therefore, asiaticoside may activate the cAMP/PKA signaling pathway to inhibit NF- $\mathrm{B}$ - and NLRP3-related inflammation. Moreover, phosphorylation of the cAMP-responsive element-binding protein at serine 133 and the expression of brain-derived neurotrophic factor were increased after asiaticoside administration. Collectively, the present results suggested that asiaticoside may play a vital role as an antidepressant and anti-inflammatory agent in the CMS mouse model by regulating the cAMP/PKA signaling pathway.

\section{Introduction}

Depression is speculated to be a stress-related mental disorder caused by mental and social burdens (1). While the precise mechanisms underlying depression remain unknown, these will most likely involve molecular and cellular abnormalities that interact with genetic and environmental factors, such as abnormal metabolism of monoamine neurotransmitters, neurogenesis and hypothalamic-pituitary-adrenal axis dysfunction (2,3). Although depression is characterized by multiple systemic manifestations, inflammation is the key event associated with these disturbances in the brain $(4,5)$. It has been shown that sustained stress leads to the activation of inflammatory signaling and increases neuronal susceptibility to damage $(6,7)$. Moreover, inflammatory cytokines impair neurogenesis, activity of neurotrophic factors and neuronal plasticity $(8,9)$. Inflammatory cytokines have been proposed to regulate monoamine neurotransmitter metabolism by inhibiting neurotransmitter synthesis and promoting monoamine neurotransmitter hydrolysis $(10,11)$. Therefore, inhibiting inflammation may be a potential therapeutic strategy for depression $(12,13)$.

Nuclear factor (NF)- $\kappa \mathrm{B}$ and nod-like receptor protein 3 (NLRP3) inflammasome are two archetypal molecular drivers 
of the inflammatory response (14). NF- $\kappa \mathrm{B}$ is a critical transcription factor that induces the expression of inflammatory mediators, such as interleukin (IL)-1 $\beta$, IL-6 and tumor necrosis factor $\alpha(\mathrm{TNF}-\alpha)$, and upregulates NLRP3 synthesis (14). Dysregulated NF- $\mathrm{B}$ signaling has been reported to be associated with numerous inflammatory, autoimmune and nervous system diseases (14). The NLRP3 inflammasome consists of a NLRP3 pattern recognition receptor, an apoptosis-associated speck-like protein (ASC) adaptor and the effector protein caspase-1. Once activated, NLRP3 interacts with the ASC adaptor protein and cleaves pro-caspase-1 to produce mature caspase-1, which activates the processing of IL-1 $\beta$ to engage the immune defense mechanism (15). Furthermore, NF- $\mathrm{BB}$ - and NLRP3-induced inflammation may play an important role in depression, Alzheimer's disease, Parkinson's disease and diabetes $(16,17)$.

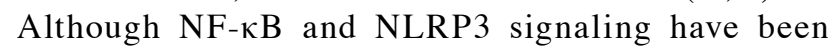
reported to contribute to inflammation, pathways such as the cAMP/protein kinase A (PKA) signaling pathway may also play important roles in regulating NF- $\kappa \mathrm{B}$ and NLRP3-related inflammatory responses (18-22). Previous studies have revealed the anti-inflammatory role of cAMP, a prototypical second messenger, which interferes with the immune response at multiple levels (18-22). Furthermore, binding of cAMP to the NLRP3 NOD-binding domain has been previously identified (22). In addition, the prototypical cAMP effector kinase PKA, a serine/threonine kinase, regulates several pathways involved in controlling inflammation by modulating gene transcription and post-translational modification of effector proteins, including NF- $\mathrm{B}$ and NLRP3 (22). Moreover, PKA upregulation may also increase the phosphorylation of cAMP-responsive element-binding protein (CREB), which is an important transcription factor involved in several critical functions in depression (23). Suppressing CREB signaling in the hippocampus has been reported to induce depressive-like behavior by interfering with the expression of CRE-regulated genes, such as brain-derived neurotrophic factor (BDNF), which plays an important role in depression (24).

Asiaticoside is one of the triterpenoid components found in Centella asiatica (L.) (25-28). It has been reported that asiaticoside exhibits anti-inflammatory, antioxidative, wound-healing, hepatoprotective and antitumor properties (25). Asiaticoside has also been shown to exert a strong anti-inflammatory effect in animal models of osteoarthritis, spinal cord injury and peritonitis (26). Moreover, asiaticoside may exert beneficial effects in central nervous system (CNS) disorders, including Parkinson's disease and dementia (27). It has been reported that the antidepressant properties of asiaticoside are mediated via activation of the BDNF/tropomyosin receptor kinase B (TrkB) signaling pathway in the chronic unpredictable mild stress (CMS) mouse model (28). However, the mechanism underlying asiaticoside-mediated alterations in BDNF signaling is yet to be elucidated. A previous study showed the neuroprotective effect of asiaticoside on Neuro-2a cells, which was related to elevation of the cAMP/CREB signal (29). In line with these previous results, it was hypothesized that the antidepressant-like properties and anti-inflammatory effects of asiaticoside may be, at least partly, via activation of the cAMP/PKA signaling pathway. The present study aimed to investigate whether asiaticoside inhibits inflammation in a mouse model of depression, and to identify the underlying mechanisms regulating the cAMP/PKA pathway.

\section{Materials and methods}

Drugs and reagents. Asiaticoside (purity $>98 \%$ ) was purchased from Nanjing Zelang Medical Technology Co., Ltd. Fluoxetine (FLX) hydrochloride was purchased from Changzhou Siyao Pharmaceuticals Co., Ltd. All other chemicals and reagents used were of analytical grade.

Animals. In total, 60 male ICR mice (age, 2 months old; weight, 18-22 g) were purchased from The Experimental Animal Center in Jiangsu Province. Animals were randomly divided into five treatment groups ( $\mathrm{n}=12 \mathrm{mice} / \mathrm{group})$ and housed in cages at room temperature $\left(22 \pm 2^{\circ} \mathrm{C}\right)$ under a 12 -h light/dark cycle (lights on at 8:00 a.m.), with ad libitum access to food and water. The mice were allowed to acclimate for 1 week before the experiment commenced. The groups were as follows: i) Normal control group; ii) CMS model group; iii) CMS + FLX (20 mg/kg, i.g) group; iv) CMS + asiaticoside $(20 \mathrm{mg} / \mathrm{kg}$, i.g) group; and v) CMS + asiaticoside (40 mg/kg, i.g) group. Asiaticoside and FLX were prepared in double-distilled water. Mice were exposed to the CMS stimuli for 4 weeks (weeks 1-4), followed by 4 weeks (weeks 5-8) of asiaticoside or FLX treatment during which CMS stimulation continued. For the control and CMS model groups, mice were given an equal volume of double-distilled water. Asiaticoside doses were selected based on a previous study (28). All experiments were conducted as per the Guidelines of the Institutional Animal Care and Use Committee of China and the present study was approved by Experimental Animal Ethics Committee of Xuzhou Medical University (Lianyungang, China).

Behavioral tests were performed after the last administration of asiaticoside or FLX (Fig. 1). CMS was introduced as previously described $(17,30)$ with minor modifications (the order of stressors was random). The normal control group animals were left undisturbed in their cages in a separate room throughout the 8 weeks of treatment. The other four groups were individually housed and subjected to a variety of stresses for 8 weeks: i) Soiled cage (200 ml water in $100 \mathrm{~g}$ sawdust bedding; ii) foreign object exposure; iii) light/dark perversion; iv) physical restraint for $2 \mathrm{~h}$; v) food deprivation for $24 \mathrm{~h}$; vi) water deprivation for $24 \mathrm{~h}$; vii) overnight illumination; viii) cage tilt $\left(45^{\circ}\right)$ for $10 \mathrm{~h}$; ix) white noise; x) 3-min oscillation; and xi) 1-min tail pinch (1 cm from the root of the tail). All the stressors were applied randomly to ensure the unpredictability of the experiment.

Sucrose preference test. Sucrose preference test was conducted every week during the experimental period (17). Mice were first deprived of water and food for $10 \mathrm{~h}$ before the test, and were then allowed to freely choose between two bottles (one with tap water, and another with $1 \%$ sucrose solution) for $10 \mathrm{~h}$. To prevent possible effects of side preference on drinking behavior, the positions of the bottles were switched after $5 \mathrm{~h}$. The consumption of the sucrose solution and tap water was estimated by weighing the bottles. Sucrose preference was calculated as sucrose preference $(\%)=$ sucrose intake $(\mathrm{g}) /[$ sucrose intake $(\mathrm{g})+$ water intake $(\mathrm{g})]$ x100.

Tail suspension test (TST). TST was performed as described previously (17). Individual mice were acoustically and visually isolated, and suspended $\sim 50 \mathrm{~cm}$ above the floor by placing adhe- 


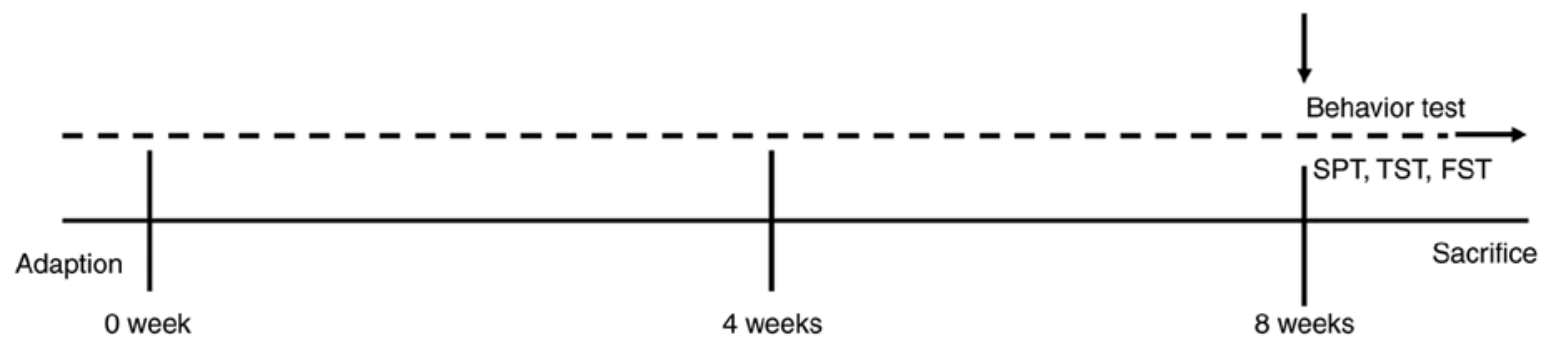

\begin{tabular}{|c|c|c|}
\hline Group I & Non-CMS & Non-CMS \\
\hline Group II & \multirow{4}{*}{ CMS } & CMS \\
\hline Group III & & $\mathrm{CMS}+\mathrm{FLX}(10 \mathrm{mg} / \mathrm{kg})$ \\
\hline Group IV & & $\mathrm{CMS}+$ Asiaticoside $(20 \mathrm{mg} / \mathrm{kg})$ \\
\hline Group V & & CMS+ Asiaticoside $(40 \mathrm{mg} / \mathrm{kg})$ \\
\hline
\end{tabular}

Figure 1. Schematic representation of the experimental procedure for CMS and treatments in mice. SPT, sucrose preference test; TST, tail suspension test; FST, forced swimming test; CMS, chronic unpredictable mild stress; FLX, fluoxetine.

sive tape $\sim 1 \mathrm{~cm}$ from the tail tip. Mice were considered immobile when they were passively suspended and remained completely motionless. Each animal was suspended for $6 \mathrm{~min}$, and total immobility was recorded during the last $4 \mathrm{~min}$ of the test.

Forced swimming test (FST). FST was carried out as described previously on the day after TST (17). Mice were forced to swim in a cylinder $(20 \mathrm{~cm}$ height $\mathrm{x} 14 \mathrm{~cm}$ diameter) containing fresh water $\left(25 \pm 1^{\circ} \mathrm{C}\right)$ to a height of $10 \mathrm{~cm}$. The immobility time was recorded as the time the mice spent floating in the water without struggling or only making movements necessary to keep their heads above the water. Each animal was forced to swim for $6 \mathrm{~min}$, and total immobility time was recorded during the last $4 \mathrm{~min}$ of the test.

Collection of tissue samples. Mice were sacrificed after the final behavioral tests were concluded. Ketamine and xylazine (100 and $10 \mathrm{mg} / \mathrm{kg}$, respectively) were intraperitoneally injected to anesthetize the mice and the mice were then sacrificed by decapitation. The whole hippocampus was rapidly dissected on an iced-plate and weighed. The hippocampus was identified as described in Paxinos and Watson's Atlas (30).

Determination of 5-hydroxytryptamine (5-HT) and norepinephrine (NE) levels. The levels of 5-HT and NE in the mouse hippocampus were determined by high-performance liquid chromatography (HPLC). The hippocampus was homogenized in extract solution, which consisted of 0.1 mM EDTA and $0.1 \mathrm{M} \mathrm{HClO}_{4}$ buffer, and the mixture was centrifuged at a speed of $20,000 \mathrm{x} \mathrm{g}$ for $30 \mathrm{~min}$ at $4^{\circ} \mathrm{C}$. Then, $50 \mu \mathrm{l}$ of the resulting supernatant was injected into the liquid chromatography system equipped with a reversed phase C18 column $(2.2 \mu \mathrm{m}, 120 \AA$ A, 2.1x100 mm; Dionex; Thermo Fisher Scientific, Inc.). The mobile phase was composed of solutions A ( $0.1 \%$ formic acid, $\mathrm{v} / \mathrm{v})$ and $\mathrm{B}$ (acetonitrile), with a gradient elution as follows: 0-6 $\mathrm{min}, 90-65 \% \mathrm{~A}$; 6-8 $\mathrm{min}, 65-0 \% \mathrm{~A}$; $8-10 \mathrm{~min}, 0 \% \mathrm{~A}$. The flow rate was maintained at $0.4 \mathrm{ml} / \mathrm{min}$, and the column temperatures were maintained at $30^{\circ} \mathrm{C}$, and was detected by ESA Coulochem ${ }^{\circledR}$ III Electrochemical Detector (Dionex; Thermo Fisher Scientific, Inc.). The detector was set at $350 \mathrm{mV}$. The identification and purity were evaluated by the chromatographic peaks as well as their quantitative evaluation, which was measured by comparing their retention times and peak areas with those of standard solutions. 5-HT (cat. no. H9523) and NE (cat. no. N-069-1ML) standards were purchased from Sigma-Aldrich (Merck KGaA).

Determination of IL-1 $\beta, I L-6, T N F-\alpha$ and cAMP levels in the hippocampus. Cytokine and cAMP concentrations in the mouse hippocampus were measured by ELISA, according to the manufacturer's instructions. The following kits were used: Mouse IL-1 $\beta$ ELISA kit (cat. no. 1210122); mouse IL-6 ELISA kit (cat. no. 1210602); mouse TNF- $\alpha$ ELISA kit (cat. no. 1217202; all purchased from Dakewei Biotechnology Co., Ltd.); and mouse cyclic adenosine monophosphate, cAMP GENLISA $^{\mathrm{TM}}$ ELISA kit (cat. no. KLM0071; Krishgen BioSystems). The concentrations were measured using a microplate reader (450 $\mathrm{nm}$ absorbance). The results were reported in picograms per milliliter $(\mathrm{pg} / \mathrm{ml})$.

Protein extraction and western blotting. Protein extracts were obtained by homogenizing hippocampal tissue in lysis buffer [50 mM Tris $\mathrm{HCl}(\mathrm{pH}$ 7.2) containing $1 \%$ sodium deoxycholate, $1 \%$ NP-40, $0.15 \mathrm{mM} \mathrm{NaCl}$ and $0.1 \%$ SDS; Roche Applied Science). A bicinchoninic acid assay was used to determine protein concentrations (Sigma-Aldrich; Merck KGaA). Equal amounts of protein $(30 \mu \mathrm{g} / \mu \mathrm{l})$ were separated by SDS-PAGE on $10 \%$ gels and were then transferred to PVDF membranes ( $0.2 \mu \mathrm{m}$; EMD Millipore). The membranes were blocked for $2 \mathrm{~h}$ with 5\% non-fat dry milk at room temperature, then incubated at $4^{\circ} \mathrm{C}$ overnight with the following antibodies: Rabbit anti-phosphorylated (p)NF-кBp65 ${ }^{\text {Ser536 }}$ (1:1,000; cat. no. orb501609; Biorbyt Ltd.), rabbit anti-NF-kBp65 (1:1,000; cat. no. orb453023; Biorbyt Ltd.), rabbit anti-NLRP3 (1:1,000; cat. no. orb101128; Biorbyt Ltd.), rabbit anti-caspase-1 (1:1,000; cat. no. ab179515; Abcam), rabbit anti-PKA (1:1,000; cat. no. ab75991; Abcam), 

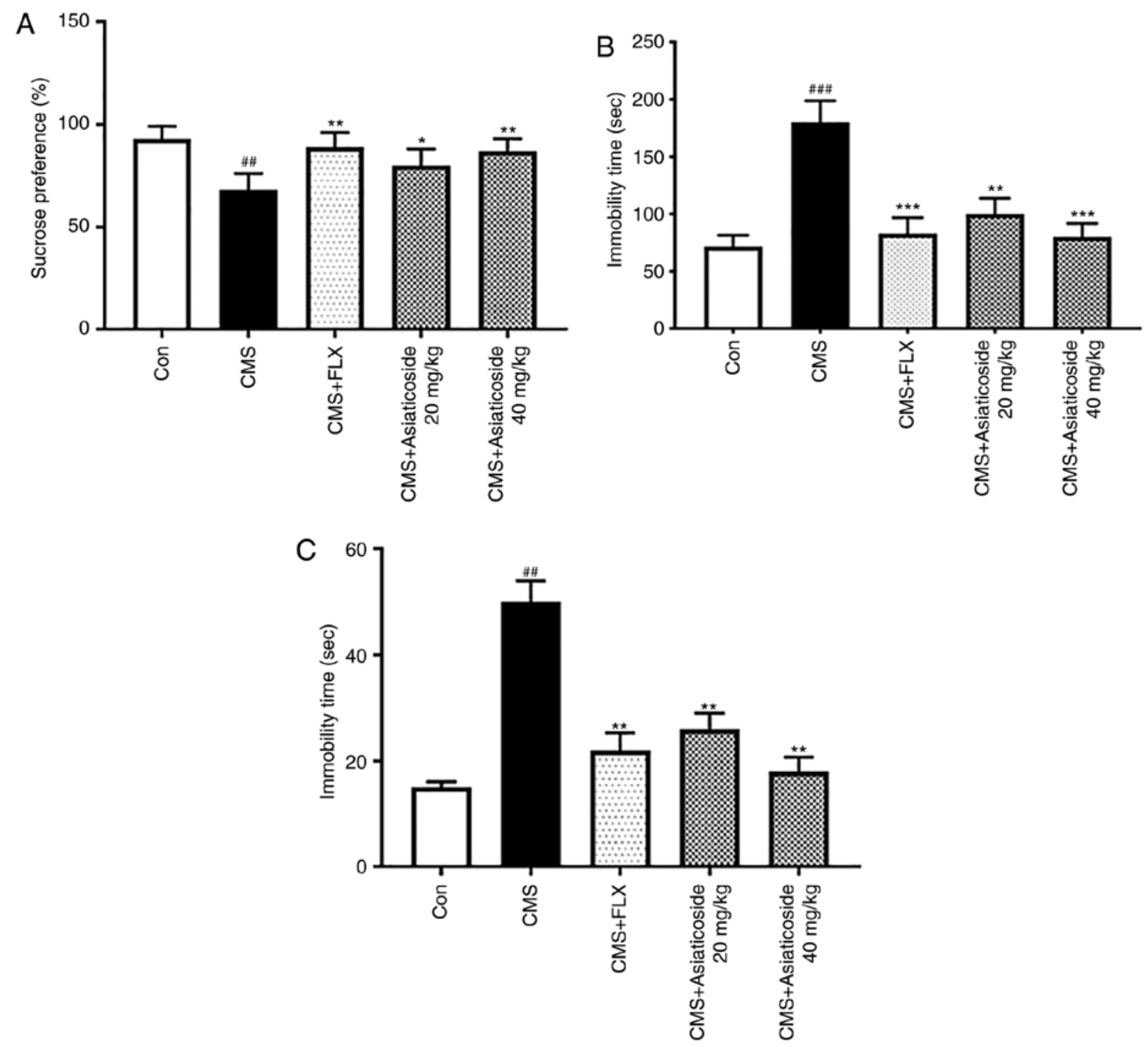

Figure 2. Effect of asiaticoside on sucrose preference. (A) Sucrose preference test was performed $24 \mathrm{~h}$ after food-and water-deprivation. Immobility time in the (B) TST and (C) FST in mice with or without CMS. Intragastric administration of the drug or vehicle was performed once a day for 4 weeks, starting from the 4th week of CMS stimulation. Immobility duration was measured in the last $4 \mathrm{~min}$ of the TST or FST. Data are presented as the mean \pm SEM. ${ }^{\# /} \mathrm{P}<0.01$,

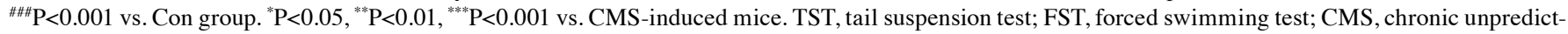
able mild stress; FLX, fluoxetine; Con, control.

rabbit anti-p-VASP ${ }^{\text {ser157 }}(1: 1,000 ;$ cat. no. ab47268; Abcam) and rabbit anti-VASP (1:1,000; cat. no. ab205952; Abcam), rabbit anti-pCREB $^{\text {Ser133 }}$ (1:1,000; cat. no. ab32096; Abcam), rabbit anti-CREB (1:1,000; cat. no. ab32515; Abcam), rabbit anti-BDNF (1:1,000; cat. no. ab108319; Abcam) and rabbit anti- $\beta$-actin (1:1,000; cat. no. ab8227; Abcam). After three washes in TBS-Triton X-100 (1\%) buffer, the membranes were incubated for $1 \mathrm{~h}$ at $24^{\circ} \mathrm{C}$, with horseradish peroxidase-labeled anti-rabbit IgG (1:5,000; cat. no. BS13271; Bioworld Technology, Inc.). Densitometric measurements were performed using the ECL Western detection system (EMD Millipore) and the Quantity One imaging program (v7.1; Bio-Rad Laboratories, Inc.).

Statistical analyses. Experiments were repeated three times. Data are presented as the mean \pm SEM. Statistical significance was assessed by one-way ANOVA followed by Tukey's test. All statistical analyses were performed using GraphPad Prism 8 software (GraphPad Software, Inc.). $\mathrm{P}<0.05$ was considered to indicate a statistically significant difference.

\section{Results}

Effects of asiaticoside on sucrose preference, TST and FST. Sucrose preference in mice is considered a measure of anhedonia (17). It was revealed that the CMS model mice exhibited significantly reduced sucrose consumption compared with the control group $(\mathrm{P}<0.01 ;$ Fig. $2 \mathrm{~A})$. Moreover, asiaticoside (20 and $40 \mathrm{mg} / \mathrm{kg}$ ) or FLX $(10 \mathrm{mg} / \mathrm{kg}$ ) administration significantly reversed the decreased sucrose consumption in the CMS model group $(\mathrm{P}<0.05, \mathrm{P}<0.01$, respectively). It was also demonstrated that CMS significantly increased the immobility time during TST ( $\mathrm{P}<0.001$; Fig. 2B) and FST ( $\mathrm{P}<0.01$; Fig. $2 \mathrm{C})$. Asiaticoside (20 and $40 \mathrm{mg} / \mathrm{kg})$ or FLX $(10 \mathrm{mg} / \mathrm{kg})$ treatment significantly reversed the increase in the immobility time in both TST $(\mathrm{P}<0.01, \mathrm{P}<0.001$, respectively; Fig. $2 \mathrm{~B})$ and FST $(\mathrm{P}<0.01$; Fig. 2C).

Effects of asiaticoside on neurotransmitters in the hippocampus. The present study also examined the levels of 

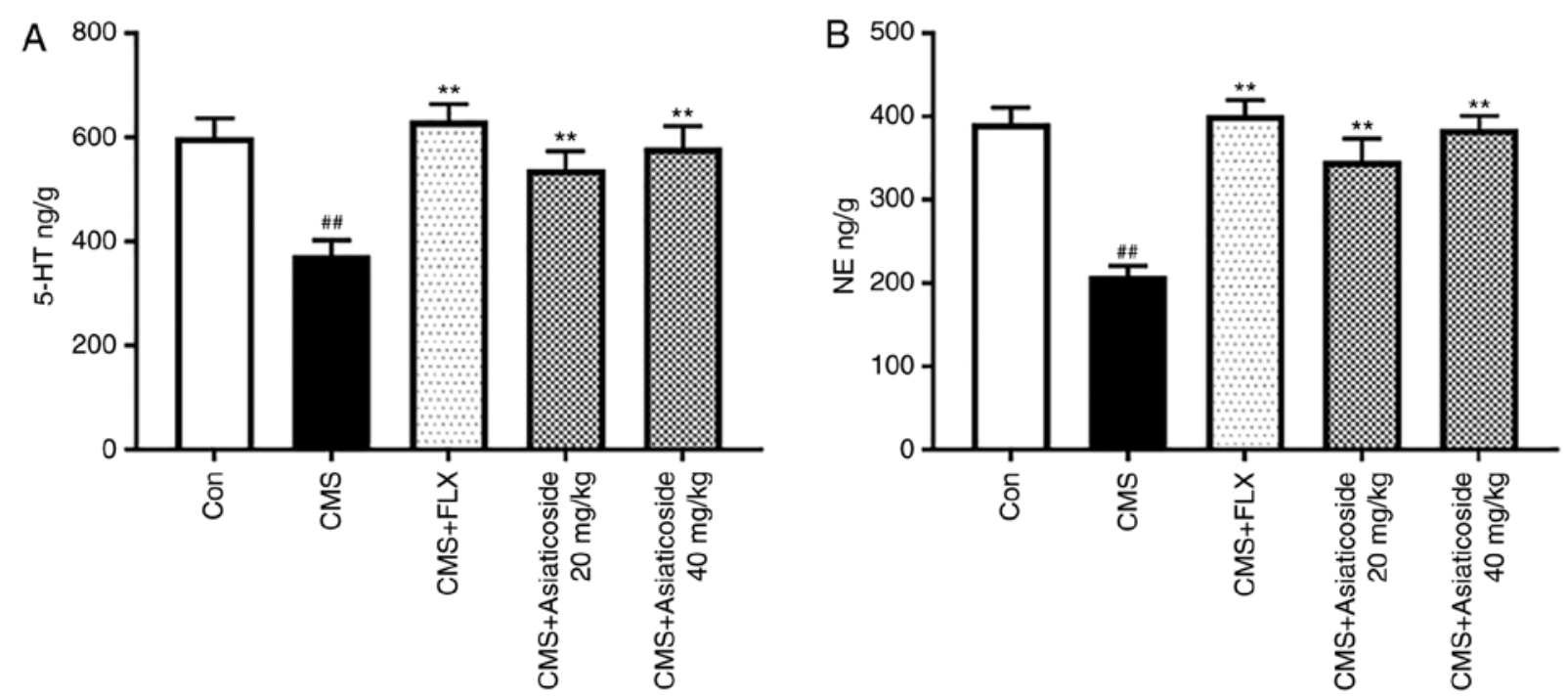

Figure 3. Effects of asiaticoside treatment on 5-HT and NE in the hippocampus of the CMS-induced mice. The hippocampus was dissected out immediately after the behavioral tests and levels of (A) 5 -HT and (B) NE were measured. Data are presented as the mean \pm SEM. ${ }^{\# /} \mathrm{P}<0.01$ vs. Con group; ${ }^{* *} \mathrm{P}<0.01$ vs. CMS-induced mice. CMS, chronic unpredictable mild stress; FLX, fluoxetine; Con, control; 5-HT, 5-hydroxytryptamine; NE, norepinephrine.
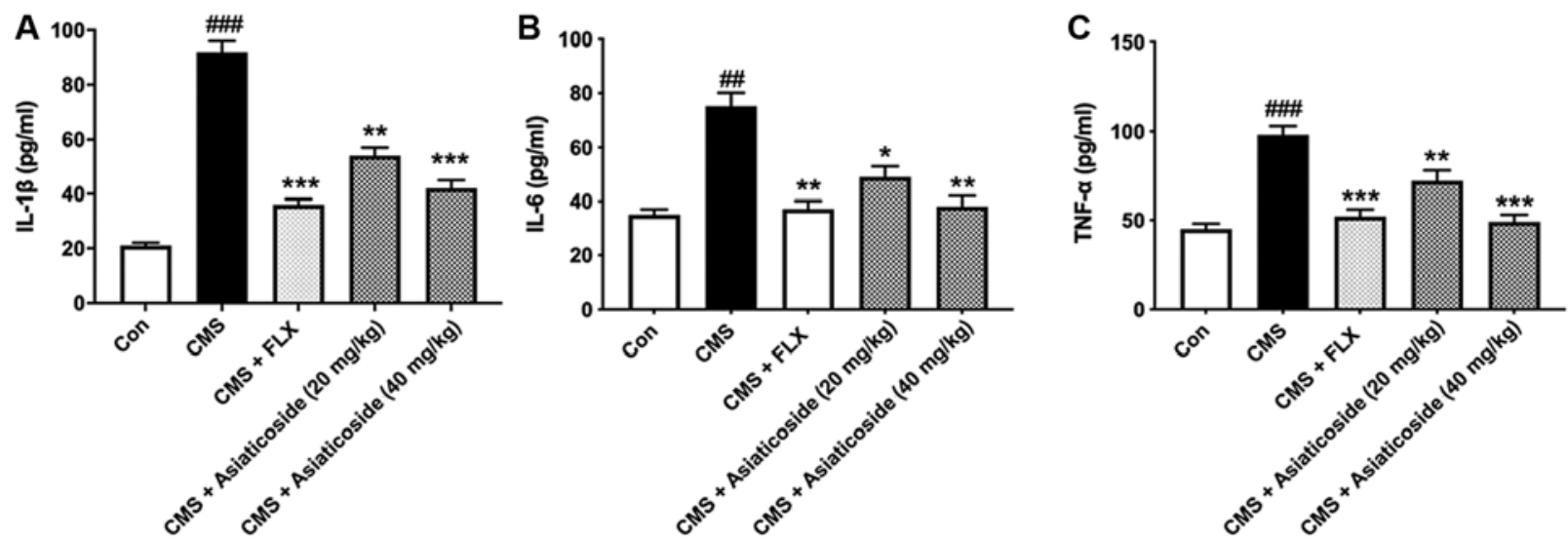

Figure 4. Effects of asiaticoside treatment on IL-1 $\beta$, IL-6 and TNF- $\alpha$ levels. (A) IL-1 $\beta$, (B) IL-6 and (C) TNF- $\alpha$ were detected using ELISA and measured using a microplate reader at $450 \mathrm{~nm}$ absorbance. Data are presented as the mean $\pm \mathrm{SEM}$. ${ }^{\# \#} \mathrm{P}<0.01,{ }^{\# \# "} \mathrm{P}<0.001$ vs. Con group; ${ }^{*} \mathrm{P}<0.05,{ }^{* * *} \mathrm{P}<0.01,{ }^{* * *} \mathrm{P}<0.001 \mathrm{vs}$. CMS-induced mice. CMS, chronic unpredictable mild stress; FLX, fluoxetine; Con, control; IL, interleukin; TNF- $\alpha$, tumor necrosis factor $\alpha$.

neurotransmitters in the hippocampus. Fig. 3 shows the effect of asiaticoside on the hippocampal levels of 5-HT and NE. The present results suggested that CMS significantly decreased the levels of 5-HT and NE in the hippocampus $(\mathrm{P}<0.01)$, whereas asiaticoside or FLX administration significantly alleviated the levels of both 5-HT ( $\mathrm{P}<0.01$; Fig. 3A) and $\mathrm{NE}(\mathrm{P}<0.01$; Fig. 3B) in the hippocampus of CMS model mice.

Effects of asiaticoside on IL-1 $\beta, I L-6$ and TNF- $\alpha$ levels. The anti-inflammatory effect of asiaticoside in CMS mice was identified in the present study (Fig. 4). It was found that treatment with asiaticoside ( 20 and $40 \mathrm{mg} / \mathrm{kg}$ ) or FLX $(10 \mathrm{mg} / \mathrm{kg})$ significantly decreased the levels of IL-1 $\beta$ in CMS mice $(\mathrm{P}<0.01, \mathrm{P}<0.001$, respectively; Fig. $4 \mathrm{~A})$. In the hippocampus of the CMS mice, IL-6 and TNF- $\alpha$ levels were significantly higher compared with the control group mice $(\mathrm{P}<0.01, \mathrm{P}<0.001$, respectively). Asiaticoside (20 and $40 \mathrm{mg} / \mathrm{kg})$ or FLX $(10 \mathrm{mg} / \mathrm{kg})$ administration significantly attenuated the increase in IL-6 $(\mathrm{P}<0.05, \mathrm{P}<0.01$, respectively; Fig. 4B) and TNF- $\alpha(\mathrm{P}<0.01, \mathrm{P}<0.001$; respectively; Fig. 4C $)$ levels in these mice.

Effects of asiaticoside on $p N F-\kappa B p 65, N L R P 3$ and caspase-1 protein expression levels. The present study measured the protein expression levels of pNF-кBp65 and NLRP3 inflammasome complex in the mice hippocampus. It was identified that the protein expression of pNF- $\mathrm{kBp} 65$ was significantly elevated $(\mathrm{P}<0.01)$ in the CMS mice (Fig. 5A). Furthermore, asiaticoside (20 and $40 \mathrm{mg} / \mathrm{kg}$ ) or FLX administration significantly reduced the expression of pNF- $\kappa B p 65(\mathrm{P}<0.05, \mathrm{P}<0.01$, respectively) in these mice. In addition, the protein expression levels of NLRP3 and mature caspase-1 (Fig. 5B) were significantly increased in the hippocampus of CMS mice $(\mathrm{P}<0.01)$. The present results also indicated that asiaticoside (20 and $40 \mathrm{mg} / \mathrm{kg})$ or FLX $(10 \mathrm{mg} / \mathrm{kg})$ treatment significantly reduced the expression levels of NLRP3 and mature caspase-1 in mice $(\mathrm{P}<0.05, \mathrm{P}<0.01$, respectively). 

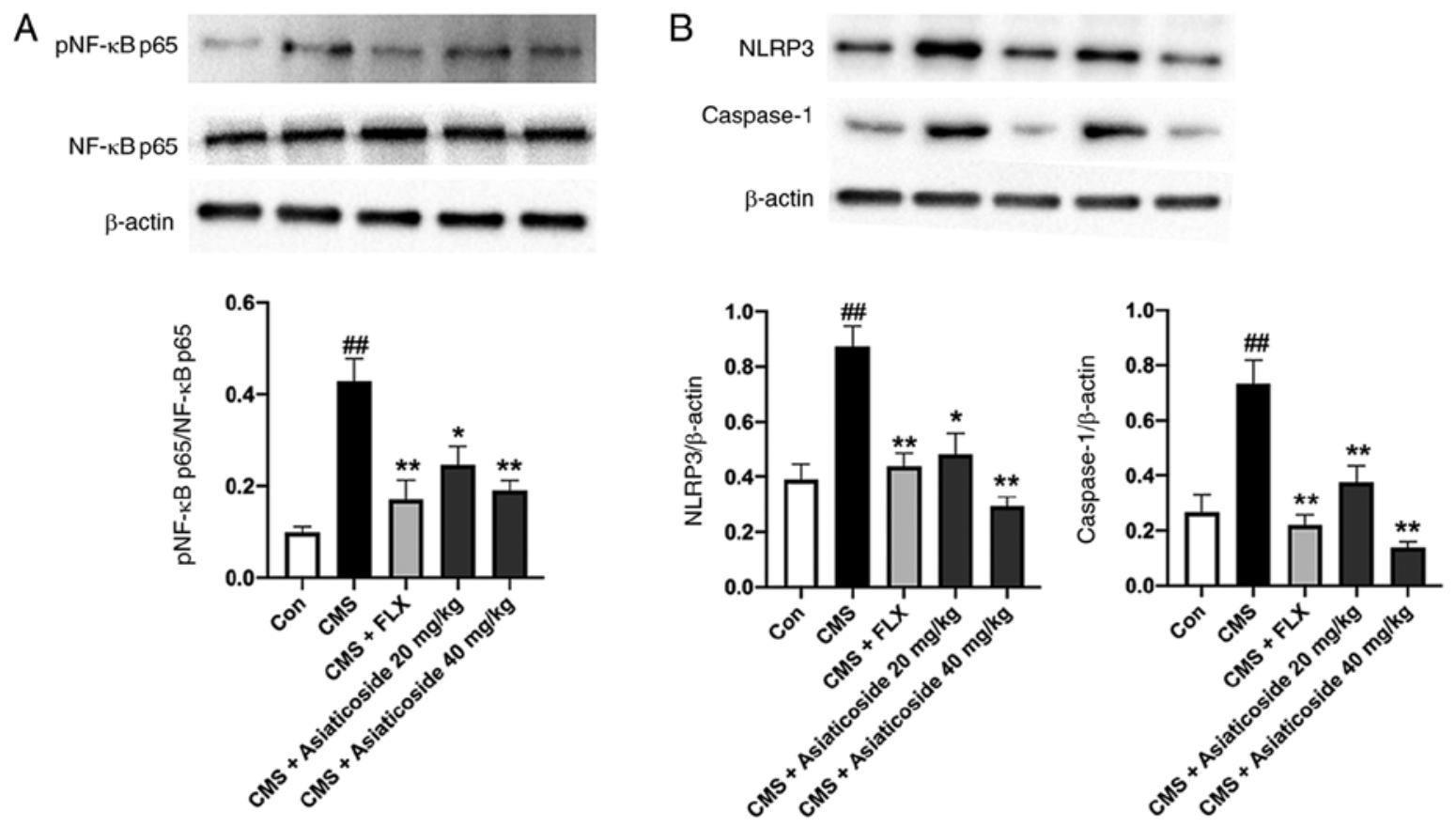

Figure 5. Effects of asiaticoside treatment on pNF-kBp65 and NLRP3 protein expression levels in the hippocampus of CMS-induced mice. The hippocampus was dissected out immediately after the behavioral tests and homogenized for western blot analysis. Expression of (A) pNF-кBp65 and (B) NLRP3 were measured. Data are presented as the mean $\pm \mathrm{SEM}$. ${ }^{\# \prime} \mathrm{P}<0.01$ vs. Con group; ${ }^{*} \mathrm{P}<0.05,{ }^{* *} \mathrm{P}<0.01$ vs. CMS-induced mice. CMS, chronic unpredictable mild stress; FLX, fluoxetine; Con, control; p, phosphorylated; NLRP3, nod-like receptor protein 3.

A

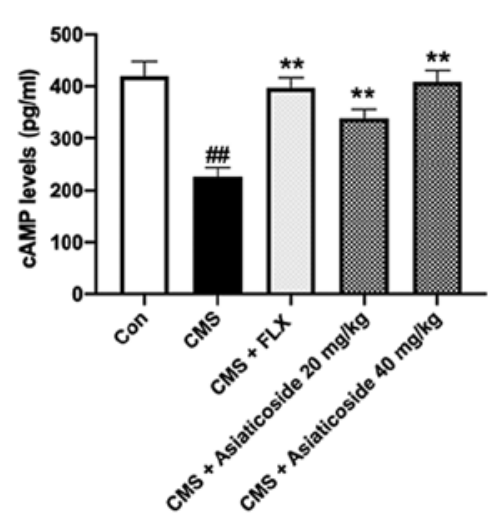

B

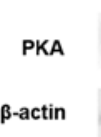

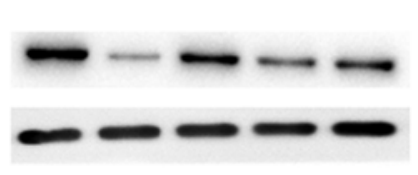

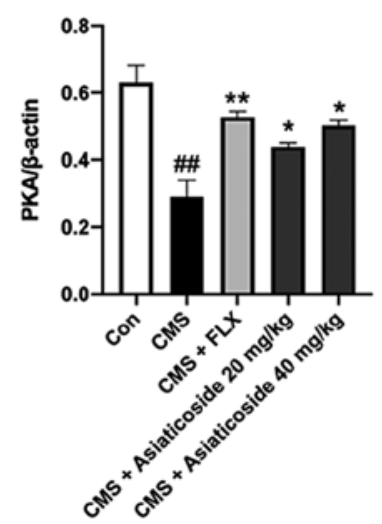

$\mathbf{C}_{\mathrm{p} w \mathrm{~s}}$
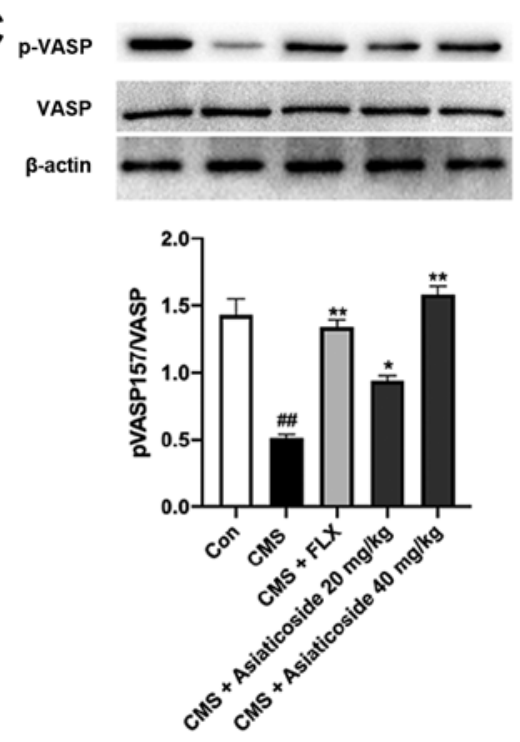

Figure 6. Effects of asiaticoside treatment on cAMP, PKA and pVASPser157 in the hippocampus of the CMS-induced mice. The hippocampus was dissected out immediately after the behavioral tests and homogenized for western blot analysis. Expression of (A) cAMP, (B) PKA and (C) pVASPser157 were measured. Data are presented as the mean \pm SEM. ${ }^{\# \#} \mathrm{P}<0.01$ vs. Con group; ${ }^{*} \mathrm{P}<0.05,{ }^{* *} \mathrm{P}<0.01$ vs. CMS-induced mice. CMS, chronic unpredictable mild stress; FLX, fluoxetine; Con, control; p, phosphorylated; PKA, protein kinase A; VASP, vasodilator-stimulated phosphoprotein

Effects of asiaticoside on cAMP, PKA and p-vasodilator-stimulated phosphoprotein $(V A S P)^{\text {serl57. }}$. The protein expression levels of cAMP, PKA and pVASP ${ }^{\mathrm{ser} 157}$ were significantly decreased in the CMS mice (Fig. 6) compared with the control group $(\mathrm{P}<0.01)$. Moreover, treatment with asiaticoside (20 and $40 \mathrm{mg} / \mathrm{kg})$ or FLX (10 mg/kg) reversed the CMS-induced decrease in the expression levels of cAMP ( $\mathrm{P}<0.01$; Fig. 6A), PKA $(\mathrm{P}<0.05, \mathrm{P}<0.01$, respectively; Fig. $6 \mathrm{~B})$ and pVASP $\mathrm{P}^{\mathrm{ser} 157}$ $(\mathrm{P}<0.05, \mathrm{P}<0.01$, respectively; Fig. $6 \mathrm{C})$. Therefore, the present results suggested that $\mathrm{CAMP/PKA}$ signaling may be involved in asiaticoside-mediated inhibition of neuroinflammation.

Effects of asiaticoside on $p C R E B^{\text {Serl33 }}$ and BDNF expression levels. It has been reported that the antidepressant-like effect of asiaticoside is mediated via the activation of BDNF signaling (29). However, the mechanism underlying asiaticoside-mediated alterations in BDNF levels is yet to be elucidated. cAMP/PKA activation has been reported to increase CREB 
A
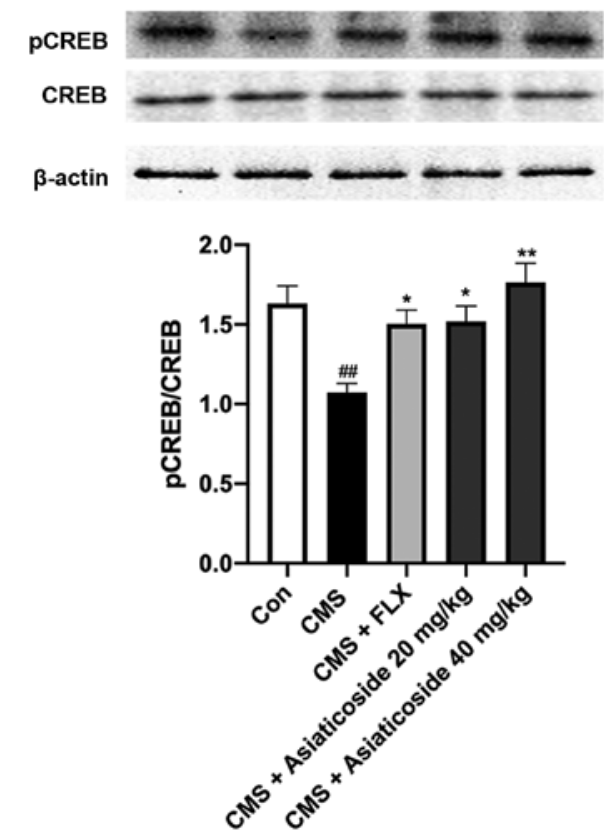

B
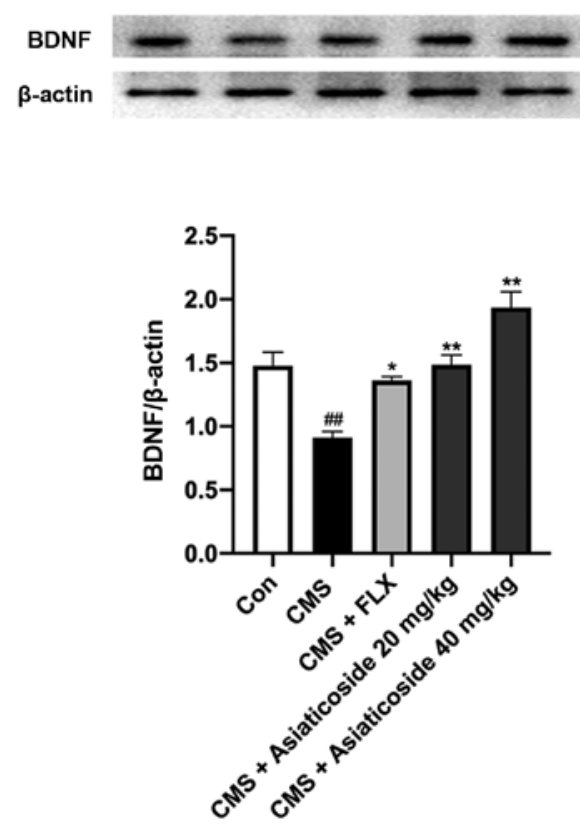

Figure 7. Effects of asiaticoside treatment on pCREB and BDNF in the hippocampus of the CMS-induced mice. The hippocampus was dissected out immediately after the behavioral tests and homogenized for western blot analysis. Expression of (A) pCREB and (B) BDNF were measured. Data are presented as the mean \pm SEM. ${ }^{\# \#} \mathrm{P}<0.01$ vs. Con group; ${ }^{*} \mathrm{P}<0.05,{ }^{* *} \mathrm{P}<0.01$ vs. CMS-induced mice. CMS, chronic unpredictable mild stress; FLX, fluoxetine; Con, control; $\mathrm{p}$, phosphorylated; CREB, cAMP-responsive element-binding protein; BDNF, brain-derived neurotrophic factor.

phosphorylation and induce the expression of CRE-regulated genes, such as BDNF (24). Therefore, the present study examined the protein expression levels of pCREB $^{\mathrm{Ser} 133}$ and BDNF in CMS mice (Fig. 7). It was found that the ratio of $\mathrm{pCREB}^{\mathrm{Ser} 133}$ to CREB was significantly decreased in the hippocampus of CMS mice $(\mathrm{P}<0.01)$, which was reversed by asiaticoside or FLX treatments $(\mathrm{P}<0.05, \mathrm{P}<0.01$, respectively; Fig. 7A). Furthermore, BDNF expression was significantly lower in the hippocampus of the CMS mice compared with the control mice $(\mathrm{P}<0.01)$. However, this effect was completely reversed upon asiaticoside or FLX treatment $(\mathrm{P}<0.01, \mathrm{P}<0.05$, respectively; Fig. 7B), thus supporting the role of asiaticoside in activating the cAMP/PKA signaling pathway.

\section{Discussion}

The present results suggested that asiaticoside administration can reverse depressive-like behavior, increase the levels of monoamine neurotransmitters, inhibit hippocampal inflammation, and ameliorate pNF-кBp65 and NLRP3 inflammasome levels in the CMS mouse model. Moreover, it was speculated that the antidepressant-like effect of asiaticoside may occur via modulation of the $\mathrm{cAMP} / \mathrm{PKA} / \mathrm{NF}-\kappa \mathrm{B} / \mathrm{NLRP} 3 / \mathrm{CREB} / \mathrm{BDNF}$ signaling pathway.

The CMS mouse model is a validated animal model of depression. CMS results in complex behavioral alterations, as well as biochemical and cellular cascades, such as neurotransmitter, endocrine, neurotrophic factors and inflammation, which are similar to those observed in patients with major depression (13). The present mouse model demonstrated decreased sucrose preference index in the sucrose preference test, and increased immobility time during TST and FST. Moreover, the present results are in line with those of previous studies using CMS mice $(17,31,32)$. It has been shown that abnormal levels of monoamine neurotransmitters are a typical feature of depression and stress in animals, and have been proposed as the principal pathogenic factor involved in this condition (10). The present results indicated that the levels of neurotransmitters, 5-HT and NE, decreased significantly after CMS exposure, which is consistent with previous studies $(3,32)$. Moreover, it was demonstrated that asiaticoside administration significantly reversed the decrease in 5-HT and NE levels in a mouse model of CMS-induced depression. In addition, it was found that CMS-induced neurobehavioral and biochemical changes could be restored by FLX, which was the positive control, and asiaticoside administration, thus indicating that asiaticoside had antidepressant effects in this model.

Previous studies revealed that inflammatory cytokines are an important risk factor in the development of depression in patients and animal models, and inhibiting inflammatory processes could ameliorate depressive symptoms $(2,31,32)$. In particular, the degradation pathways of the monoamine neurotransmitter precursors have been reported to be activated by inflammatory factors, which may lead to an increase in the levels of these neurotransmitters (10). A previous study also showed that asiaticoside exhibited a strong anti-inflammatory effect in animal models of cognitive deficits, spinal cord injury and cerebral ischemia-reperfusion injury $(27,33,34)$. In the present study, it was demonstrated that CMS increased hippocampal IL-1 $\beta$, IL- 6 and TNF- $\alpha$ levels, which were alleviated by asiaticoside administration, thus indicating that the antidepressant effect of asiaticoside may be due to its anti-inflammatory properties.

CMS has previously been reported to induce activation of NF- $\mathrm{NB}$ and the NLRP3 inflammasome, which in turn 
increased the levels of IL-1 $\beta$, IL-6 and TNF- $\alpha$ in the hippocampus of animal models of depression (16). Consistent with this previous study (16), the present results suggested that the expression levels of pNF- $\mathrm{Bp} 65$ and the NLRP3 inflammasome, which constitutes NLRP3 and caspase-1, were significantly increased in the CMS group compared with the control group. Furthermore, it was demonstrated that asiaticoside inhibited the activation of $\mathrm{pNF}-\kappa \mathrm{Bp} 65$, NLRP3 and caspase-1, leading to a reduction in the levels of inflammatory cytokines and subsequent inflammatory response. It has also been reported that asiaticoside may attenuate inflammation by downregulating $\mathrm{pNF}-\kappa \mathrm{Bp} 65$ and the levels of inflammatory cytokines, such as IL- $1 \beta$, IL- 6 and TNF- $\alpha$, in a rat model of $\beta$-amyloid-induced Alzheimer's disease (27).

The role of the cAMP/PKA signaling pathway in depression and motor activity disturbances has been previously identified (24). Moreover, studies have shown a prominent role of the cAMP/PKA signaling pathway in regulating $\mathrm{NF}-\kappa \mathrm{B}-$ and NLRP3-related inflammation (18-22). NF- $\kappa \mathrm{B}$ is a nuclear transcription factor, which is regulated by cAMP via PKA. In the dimeric form, $N F-\kappa B$ regulates the expression of genes involved in immune and inflammatory responses (18-21). Furthermore, the direct binding of cAMP to NLRP3 has also been revealed (22). Therefore, it was speculated that the cAMP/PKA signaling pathway may have an important role in suppressing inflammatory responses. Moreover, it has been shown that chronic stress inhibited cAMP/PKA signaling in animal models of depression (35-37). Consistent with previous studies, the present results indicated that CMS downregulated the expression levels of cAMP and PKA in the mouse hippocampus, whereas asiaticoside administration ameliorated these changes (35-37). In addition, phosphorylation of VASP157, a marker of PKA activation, was increased in the asiaticoside-treated mice. Therefore, the present results suggested that the antidepressant and anti-inflammatory activities of asiaticoside in the CMS mouse model may be mediated via cAMP/PKA signaling.

Exposure to chronic stress may induce depressive-like behavior in animal models, inducing some of the main features of depression, such as reduced levels of pCREB and BDNF (24). Moreover, increased PKA activation has been reported to enhance CREB phosphorylation, which regulates the transcription of several genes, including BDNF, which is involved in several critical functions of the CNS such as learning and emotional responses (24). While previous studies using senescence-accelerated mice or depression mouse models have identified the involvement of CREB or $\mathrm{BDNF} /$ TrkB signaling in regulating asiaticoside-mediated improvements in behavioral response $(28,38)$, relatively few studies have evaluated the potential role of cAMP/PKA signaling in this process. The present results suggested that CMS caused a significant reduction in pCREB and BDNF levels in the hippocampus. Furthermore, it was found that asiaticoside treatment increased pCREB and BDNF levels, and cAMP/PKA signaling in a CMS model. Based on these results, it was speculated that activation of the cAMP/PKA signaling pathway by asiaticoside may play an important role in its antidepressant effect.

In conclusion, the present results indicated that asiaticoside may have an antidepressant-like effect in the CMS mouse model. It was revealed that asiaticoside treatment increased the levels of monoamine neurotransmitters, and reversed the increase in inflammatory cytokines induced by CMS. Moreover, the antidepressant and anti-inflammatory mechanism of action of asiaticoside may be via regulation of the cAMP/PKA signaling pathway. The present study may facilitate the development of asiaticoside as a novel therapeutic for the treatment of depression. However, further studies are required to further identify the mechanism of asiaticoside in the activation of PKA signalling in depression, and to assess its clinical effectiveness in patients with depression or similar disorders. Future studies will use neurons or glial cells, astrocytes or microglia, in vivo and in vitro to further support the conclusion. Collectively, the present results suggested that administration of asiaticoside may be a useful natural adjuvant in antidepressant therapy.

\section{Acknowledgements}

Not applicable.

\section{Funding}

This study was supported by the National Natural Science Foundation of China (grant no. 81870943) and the Postdoctoral Research Fund of Xuzhou Medical University (grant no. 2015107026).

\section{Availability of data and materials}

The datasets used and/or analyzed during the current study are available from the corresponding author on reasonable request.

\section{Authors' contributions}

LW and TG made substantial contributions to acquisition of data, and analysis and interpretation of data. YG interpreted data and revised the manuscript critically for important intellectual content. YX made substantial contributions to conception and design, and agreed to be accountable for all aspects of the work in ensuring that questions related to the accuracy or integrity of any part of the work are appropriately investigated and resolved. All authors read and approved the final manuscript.

\section{Ethics approval and consent to participate}

All experiments were conducted as per the Guidelines of the Institutional Animal Care and Use Committee of China and the present study was approved by Experimental Animal Ethics Committee of Xuzhou Medical University

\section{Patient consent for publication}

Not applicable.

\section{Competing interests}

The authors declare that they have no competing interests. 


\section{References}

1. Ota KT and Duman RS: Environmental and pharmacological modulations of cellular plasticity: Role in the pathophysiology and treatment of depression. Neurobiol Dis 57: 28-37, 2013.

2. Yu HY, Yin ZJ, Yang SJ, Ma SP and Qu R: Baicalin reverses depressive-like behaviours and regulates apoptotic signalling induced by olfactory bulbectomy. Phytother Res 30: 469-475, 2016.

3. Lee WY, Jang SW, Lee JS, Kim YH, Kim HG, Han JM, Kim DW, Yi MH, Choi MK and Son CG: Uwhangchungsimwon, a traditional herbal medicine, protects brain against oxidative injury via modulation of hypothalamus-pituitary-adrenal (HPA) response in a chronic restraint mice model. J Ethnopharmacol 151: 461-469, 2014.

4. Miller AH and Raison CL: The role of inflammation in depression: From evolutionary imperative to modern treatment target. Nat Rev Immunol 16: 22-34, 2016.

5. Inserra A, Mastronardi CA, Rogers G, Licinio J and Wong ML: Neuroimmunomodulation in major depressive disorder: focus on caspase 1, inducible nitric oxide synthase, and interferon-gamma. Mol Neurobiol 56: 4288-4305, 2019.

6. Liberman AC, Trias E, da Silva Chagas L, Trindade P, Dos Santos Pereira M, Refojo D, Hedin-Pereira C and Serfaty CA: Neuroimmune and inflammatory signals in complex disorders of the central nervous system. Neuroimmunomodulation 25 246-270, 2018

7. Lanquillon S, Krieg JC, Bening-Abu-Shach U and Vedder $\mathrm{H}$ Cytokine production and treatment response in major depressive disorder. Neuropsychopharmacology 22: 370-379, 2000.

8. Jeon SW and Kim YK: The role of neuroinflammation and neurovascular dysfunction in major depressive disorder. J Inflamm Res 11: 179-192, 2018.

9. $\mathrm{Yu} \mathrm{H}$, Zhang $\mathrm{F}$ and Guan X: Baicalin reverse depressive-like behaviors through regulation SIRT1-NF-kB signaling pathway in olfactory bulbectomized rats. Phytother Res 33: 1480-1489, 2019

10. Barua CC, Haloi P, Saikia B, Sulakhiya K, Pathak DC, Tamuli S, Rizavi H and Ren X: Zanthoxylum alatum abrogates lipopolysaccharide-induced depression-like behaviours in mice by modulating neuroinflammation and monoamine neurotransmitters in the hippocampus. Pharm Biol 56: 245-252, 2018.

11. Raison CL, Capuron L and Miller AH: Cytokines sing the blues: Inflammation and the pathogenesis of depression. Trends Immunol 27: 24-31, 2006.

12. Duarte-Silva E, Macedo D, Maes M and Peixoto CA: Novel insights into the mechanisms underlying depression-associated experimental autoimmune encephalomyelitis. Prog Neuropsychopharmacol Biol Psychiatry 93: 1-10, 2019.

13. Yirmiya R, Rimmerman $\mathrm{N}$ and Reshef R: Depression as a microglial disease. Trends Neurosci 38: 637-658, 2015.

14. Afonina IS, Zhong Z, Karin M and Beyaert R: Limiting inflammation-the negative regulation of NF- $\mathrm{KB}$ and the NLRP3 inflammasome. Nat Immunol 18: 861-869, 2017.

15. Ratajczak MZ, Adamiak M, Thapa A, Bujko K, Brzezniakiewicz-Janus K and Lenkiewicz AM: NLRP3 inflammasome couples purinergic signaling with activation of the complement cascade for the optimal release of cells from bone marrow. Leukemia 33: 815-825, 2019.

16. Shao BZ, Cao Q and Liu C: Targeting NLRP3 inflammasome in the treatment of CNS diseases. Front Mol Neurosci 11: 320, 2018.

17. Yu HY, Yin ZJ, Yang SJ and Ma SP: Baicalin reverse AMPA receptor expression and neuron apoptosis in chronic unpredictable mild stress rats. Biochem Biophys Res Commun 451: 467-472, 2014.

18. Gerlo S, Kooijman R, Beck IM, Kolmus K, Spooren A and Haegeman G: Cyclic AMP: A selective modulator of NF- $\kappa$ B action. Cell Mol Life Sci 68: 3823-3841, 2011.

19. Zuo L, Shi L and Yan F: The reciprocal interaction of sympathetic nervous system and cAMP-PKA-NF-kB pathway in immune suppression after experimental stroke. Neurosci Lett 627: 205-210, 2016.

20. Gilmore TD: Introduction to NF-kappaB: Players, pathways, perspectives. Oncogene 25: 6680-6684, 2006a.
21. Gilmore TD and Herscovitch M: Inhibitors of NF-kappaB signaling: 785 and counting. Oncogene 25: 6887-6899, 2006b.

22. Mortimer L, Moreau F, MacDonald JA and Chadee K: NLRP3 inflammasome inhibition is disrupted in a group of auto-inflammatory disease CAPS mutations. Nat Immunol 17: 1176-1186, 2016.

23. Keil MF, Briassoulis G, Stratakis CA and Wu TJ: Protein kinase $\mathrm{A}$ and anxiety-related behaviors: A mini-review. Front Endocrinol (Lausanne) 7: 83, 2016.

24. Ramezany Yasuj S, Nourhashemi M, Keshavarzi S Motaghinejad M and Motevalian M: Possible role of cyclic AMP response element binding/brain-derived neurotrophic factor signaling pathway in mediating the pharmacological effects of duloxetine against methamphetamine use-induced cognitive impairment and withdrawal-induced anxiety and depression in rats. Adv Biomed Res 8: 11, 2019.

25. Gao YZ, Zhao LF, Ma J, Xue WH and Zhao H: Protective mechanisms of wogonoside against Lipopolysaccharide/ D-galactosamine-induced acute liver injury in mice. Eur $\mathrm{J}$ Pharmacol 780: 8-15, 2016.

26. He L, Hong G, Zhou L, Zhang J, Fang J, He W, Tickner J, Han X, Zhao L and Xu J: Asiaticoside, a component of Centella asiatica attenuates RANKL-induced osteoclastogenesis via NFATc1 and NF- $\kappa$ B signaling pathways. J Cell Physiol 234: 4267-4276, 2019.

27. Zhang C, Chen S, Zhang Z, Xu H, Zhang W, Xu D, Lin B and Mei Y: Asiaticoside alleviates cerebral ischemia-reperfusion injury via NOD2/mitogen-activated protein kinase (MAPK)/nuclear factor kappa B (NF- $\mathrm{B}$ ) signaling pathway. Med Sci Monit 26: e920325, 2020.

28. Luo L, Liu XL, Mu RH, Wu YJ, Liu BB, Geng D, Liu Q and Yi LT: Hippocampal BDNF signaling restored with chronic asiaticoside treatment in depression-like mice. Brain Res Bull 114: 62-69, 2015.

29. Nalinratana N, Meksuriyen D and Ongpipattanakul B: Differences in neuritogenic activity and signaling activation of madecassoside, asiaticoside, and their aglycones in neuro-2a cells. Planta Med 84: 1165-1173, 2018.

30. Paxinos G and Watson C: The Rat Hippocampus in Stereotaxic Coordinates. Academic Press, San Diego, CA, 1998.

31. Du RH, Tan J, Sun XY, Lu M, Ding JH and Hu G: Fluoxetine inhibits NLRP3 inflammasome activation: Implication in depression. Int J Neuropsychopharmacol 19: 19, 2016.

32. Deng XY, Li HY, Chen JJ, Li RP, Qu R, Fu Q and Ma SP: Thymol produces an antidepressant-like effect in a chronic unpredictable mild stress model of depression in mice. Behav Brain Res 291: 12-19, 2015.

33. Chen S, Yin ZJ, Jiang C, Ma ZQ, Fu Q, Qu R and Ma SP: Asiaticoside attenuates memory impairment induced by transient cerebral ischemia-reperfusion in mice through anti-inflammatory mechanism. Pharmacol Biochem Behav 122: 7-15, 2014.

34. Luo Y, Fu C, Wang Z, Zhang Z, Wang H and Liu Y: Asiaticoside attenuates the effects of spinal cord injury through antioxidant and anti-inflammatory effects, and inhibition of the p38-MAPK mechanism. Mol Med Rep 12: 8294-8300, 2015.

35. Li XY, Qi WW, Zhang YX, Jiang SY, Yang B, Xiong L and Tong JC: Helicid ameliorates learning and cognitive ability and activities cAMP/PKA/CREB signaling in chronic unpredictable mild stress rats. Biol Pharm Bull 42: 1146-1154, 2019.

36. Zhang R, Guo L, Ji Z, Li X, Zhang C, Ma Z, Fu Q, Qu R and Ma S: Radix scutellariae attenuates CUMS-induced depressive-like behavior by promoting neurogenesis via cAMP/PKA pathway. Neurochem Res 43: 2111-2120, 2018.

37. Jiang H, Zhang X, Wang Y, Zhang H, Li J, Yang X, Zhao B, Zhang $\mathrm{C}, \mathrm{Yu} \mathrm{M}, \mathrm{Xu} \mathrm{M}$, et al: Mechanisms underlying the antidepressant response of acupuncture via PKA/CREB signaling pathway. Neural Plast 2017: 4135164, 2017.

38. Lin X, Huang R, Zhang S, Wei L, Zhuo L, Wu X, Tang A and Huang Q: Beneficial effects of asiaticoside on cognitive deficits in senescence-accelerated mice. Fitoterapia 87: 69-77, 2013.

This work is licensed under a Creative Commons Attribution-NonCommercial-NoDerivatives 4.0 International (CC BY-NC-ND 4.0) License. 